\title{
Challenges and Approaches in Modeling Wind Power Plants in Electric Power Systems
}

\author{
Aleksey Suvorov ${ }^{1, a)}$, Igor Razzhivin ${ }^{1, b)}$ and Arina Evseeva ${ }^{1, c)}$ \\ ${ }^{1}$ National Research Tomsk Polytechnic University, 634050 Tomsk, Russia. \\ a)Corresponding author: suvorovaa@tpu.ru \\ b)lionrash@tpu.ru \\ c)evseeva@tpu.ru
}

\begin{abstract}
Wind power is a rapidly developing area in the global energy industry. Wind power plants (WPP) with a capacity from several kilowatts to megawatts are introduced in many countries of the world. when the share of electrical energy generated by WPP is relatively small in the electric power system (EPS), in emergency mode, relay protection and automation can turn off such WPP without any special influence on the EPS. However, today the installed capacity of individual wind farms in different countries reaches several hundred megawatts, and various changes in operating modes, including accidents in such cases, can not affect the operation mode of the EPS. The issues of modeling WPP are relevant for energy. However, the complexity of mathematical models and the specifics of mathematical modeling do not allow assessing the impact of WPP on EPS. The article discusses the main assumptions in modeling.
\end{abstract}

\section{INTRODUCTION}

Wind power is developing dynamically in many countries of Europe and America. Today, China, USA, Brazil, India, Germany, Denmark, Great Britain are recognized as leaders in wind power [1]. The global wind power industry made a profit and exists without any subsidies, but in the conditions of active state regulation and in some countries successfully competes with traditional energy.

However, the use of wind energy has its own challenges. The wind is intermittent, it is uncontrolled and can change its direction and speed during the day. As a result, the change or disturbance of the wind flow is reflected in the change in the actual output power of each wind turbine (WT) within the wind power plant (WPP). While small wind turbines are installed in the electric power system (EPS), it is no affect the operation of the EPS; however, when the share of wind turbines becomes a significant high level, the effect of wind energy becomes noticeable and requires separate study. Features and specific functioning of the WT equipment significantly change and affect the processes and dynamic properties of the EPS, power equipment operating conditions, relay protection, emergency and process automation. Many factors affect the sustained operation of the WPP itself in an EPS, as well as the WPP have an impact on the operating modes of the EPS. Due to the intensive development of wind energy, there is an inevitable need to study the operation of WT and the detailed modeling of their work under different wind loads, and with all of modes of their work, as normal, emergency and post-emergency. The well-known specificity and complexity of modern EPSs exclude the possibility of obtaining all the necessary information, especially of an emergency nature, by natural means and through physical modeling. Therefore, the main method of obtaining it is mainly mathematical modeling $[2,3]$.

\section{APPROACHES IN MODELING}

WT manufacturers have developed a mathematical models for evaluating the electrical and mechanical behavior of WT with the highest level of accuracy, but detailed models from manufacturers are not suitable for studying the 
stability of large power systems due to the large amount of input data and the high complexity of computational operations, and the use of such models in scientific limited privacy of information.

In order to solve these problems, the International Electrotechnical Commission (IEC) has been developed IEC 61400-27 standard [4], relating to determining the total (i.e., simplified or standard) dynamic models for wind turbines. According to the provisions of the standard and IEEE (Institute of Electrical and Electronics Engineers) to classify the type of wind turbine electrical machine as follows (Fig. 1):

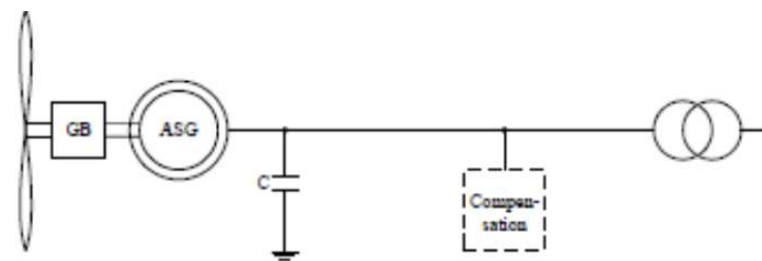

(a)

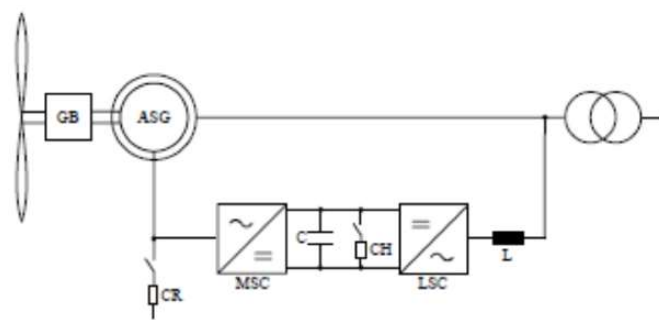

(c)

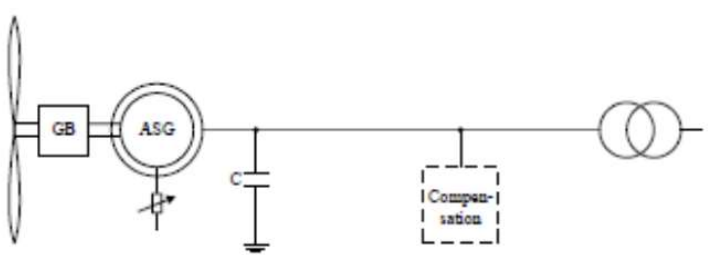

(b)

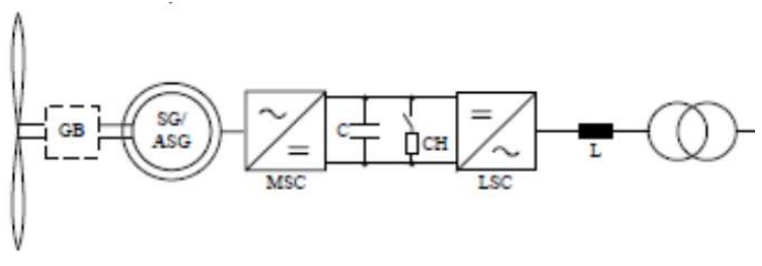

(d)

FIGURE 1. The overall structure of WT models

a) Type 1: Fixed-speed with a squirrel cage induction generator (SCIG);

b) Type 2: Semi-variable speed with a wound rotor induction generator (WRIG);

c). Type 3: Semi-variable speed with double fed induction generator (DFIG);

d) Type 4: Full-variable speed with permanent magnet synchronous generator (PMSG), SCIG, wound rotor synchronous generator (WRSG)

Depending on the purpose of modeling the above-mentioned concepts of WT in the EPS, a large number of different software tool (ST) are used. Each of ST is specifically designed for specific types of research. They can be divided into two groups: ST for modeling electromagnetic transients (ATP-EMTP, DCG/EPRI EMTP, EMTDCTM, SimPowerSystems (Matlab) and Simpow ${ }^{\mathrm{TM}}$ ) and ST for modeling electromechanical transients (PSS/ETM, Simpow $^{\mathrm{TM}}$, CYMSTAB, Eurostag, PowerFactory and Netomac ${ }^{\mathrm{TM}}$ ).

\section{CHALLENGES}

The implementation of a WT model in any ST is simple, although often cumbersome. However, some types of ST contain restrictions that make it impossible to physically correct the implementation of complete models. In the tools for calculating electromechanical transients, the basic algorithms of the program are traditionally based on the assumption that all electromagnetic transients are already completed and that only electromechanical transients and control processes are present in the network. Therefore, in order to include the model of a WT in the program for calculating electromechanical processes, it is necessary to adopt a number of assumptions, for example:

1. Ignoring magnetic saturation.

2. Sinusoidal flow distribution.

3. Any loss other than copper is ignored.

4. Voltages and currents of the stator of the main frequency are sinusoidal.

In addition, the following assumptions apply to some systems analyzed [6-8]:

a) in both systems with variable speed (types 3 and 4), the entire rotating mass is represented by one element, which means that the so-called concept of "one mass model" is used.

b) in both systems (types 3 and 4), with current control circuits are modeled as current sources. 
c) in a system based on a DFIG (type 3), the voltages and currents of the rotor are sinusoidal at the frequency, taking into account the slip.

d) in a type 4 , the synchronous generator does not have damping windings.

e) when using a diode rectifier in a direct drive wind turbine, the commutation is ignored.

Assumption (a) is made as the mechanical and electrical properties of wind turbines with variable speed are decoupled by power electronic converters. Thus, the properties of the shaft practically do not affect the interaction with the network, which is the main subject in studies of the power system.

Assumptions (b) and (c) are made in order to simulate the power electronics in programs for modeling electromechanical transients and are usually used in the simulation of dynamic processes in the power system. The full converter model requires a significant reduction in the time step of the simulation and the inclusion of higher harmonics in the equations describing the processes in the network.

Assumption (d) is made because the damper windings in the synchronous generator should not be taken into account. When a full scale converter is used, the rotational speed of the generator is controlled by a power converter that prevents vibrations.

When using a full scale converter, damper windings are necessary for switching. However, the commutation is neglected in accordance with assumption (e), and therefore the damper windings are also ignored.

The implementation of the model of the electric machine is often ignored electromagnetic transients. This means that the derivatives of the flows $\frac{\mathrm{d} \psi_{\mathrm{d}}}{\mathrm{dt}}, \frac{\mathrm{d} \Psi_{\mathrm{q}}}{\mathrm{dt}}$ are ignored in the stator windings, and the stator flows are calculated not as

state variables, but as algebraic variables. Implementations of this type of model are called models for calculating dynamic stability. These two model implementations are often referred to as third- and fifth-order models, respectively, where order denotes the number of state variables in the generator model [6].

The value of WT current in the third-order model is lower than in the fifth-order model. Neglecting stator flows leads to a discrepancy in the braking torque of the generator. Consequently, this leads to a discrepancy in the rotational rotor speed $\omega$. When applying the general model of the third order $\omega$ of the WT increases immediately when a shortcircuit appears. When using the model of the fifth order, the rotor speed of the generator decreases after elimination of short-circuits, which is close to the natural behavior of the EM during a short circuit [7].

\section{CONCLUSION}

Thus, when modeling WT, it is necessary to take into account existing simplifications. Moreover, such simplifications may result in negligence in the implementation of projects for WPP in the case when the simulation results will not coincide with real data, which can lead to serious consequences of the operation of an EPS in case of accidents and, as a result, financial costs.

\section{ACKNOWLEDGMENTS}

The work was supported by Ministry of Science and Higher Education of Russian Federation, according to the research project MK-2150.2019.9.

\section{REFERENCES}

1. Global Wind Energy Council, Annual Global Wind Report 2018, see https://gwec.net/global-wind-report-2018/

2. A. Askarov and N. Ruban, MATEC Web Conf. 141, 01038 (2017).

3. M.V. Andreev, A.S. Gusev, N. Yu. Ruban, A.A. Suvorov, R.A. Ufa, A.B. Askarov, J. Bemš and T. Králík, IEEE Trans on Power Syst. 34(2), 1404-1415 (2019).

4. I. Razzhivin, J. Bay, A. Kievets and A. Askarov, Elect Elect Automatica (EEA), 67(1), 54-60 (2018).

5. IEC 61400-27-1:2015 Wind turbines - Part 27-1: Electrical simulation models - Wind turbines, (2015), p. 200.

6. T. Ackermann, Wind Power in Power Systems (John Wiley \& Sons, Chichester, 2005), p.695.

7. P. Kundur, Power System Stability and Control (McGraw-Hill, New York, 1994), p. 1199.

8. T. Petru and T. Thiringer, 2000 Nordic Workshop on Power and Industrial Electronics, Aalborg, 2000, edited F. Blaabjerg by (Aalborg University, Aalborg, 2000). 